\title{
Analysis of Diaphragm Movement during Tidal Breathing and during its Activation while Breath Holding Using MRI Synchronized with Spirometry
}

\author{
P. KOLÁR̆ ${ }^{1}$, J. NEUWIRTH ${ }^{2}$, J. ŠANDA ${ }^{2}$, V. SUCHÁNEK ${ }^{2}$, Z. SVATÁ ${ }^{2}$, J. VOLEJNÍK $^{3}$, \\ M. PIVEC ${ }^{1}$
}

${ }^{1}$ Department of Rehabilitation, Second Medical Faculty, Charles University and University Hospital Motol, Prague, ${ }^{2}$ Department of Imaging Methods, Second Medical Faculty, Charles University and University Hospital Motol, Prague, ${ }^{3}$ Kurka.Med, s.r.o., Prague, Czech Republic

Received August 15, 2007

Accepted May 22, 2008

On-line July 18, 2008

\section{Summary}

Using magnetic resonance imaging (MRI) in conjunction with synchronized spirometry we analyzed and compared diaphragm movement during tidal breathing and voluntary movement of the diaphragm while breath holding. Breathing cycles of 16 healthy subjects were examined using a dynamic sequence (77 slices in sagittal plane during $20 \mathrm{~s}$, 1NSA, 240x256, TR4.48, TE2.24, FA90, TSE1, FOV 328). The amplitude of movement of the apex and dorsal costophrenic angle of the diaphragm were measured for two test conditions: tidal breathing and voluntary breath holding. The maximal inferior and superior positions of the diaphragm were subtracted from the corresponding positions during voluntary movements while breath holding. The average amplitude of inferio-superior movement of the diaphragm apex during tidal breathing was $27.3 \pm 10.2 \mathrm{~mm}$ (mean $\pm \mathrm{SD}$ ), and during voluntary movement while breath holding was $32.5 \pm 16.2$ $\mathrm{mm}$. Movement of the costophrenic angle was $39 \pm 17.6 \mathrm{~mm}$ during tidal breathing and $45.5 \pm 21.2 \mathrm{~mm}$ during voluntary movement while breath holding. The inferior position of the diaphragm was lower in 11 of 16 subjects (68.75\%) and identical in 2 of 16 (12.5\%) subjects during voluntary movement compared to the breath holding. Pearson's correlation coefficient was used to demonstrate that movement of the costophrenic angle and apex of the diaphragm had a linear relationship in both examined situations $(r=0.876)$. A correlation was found between the amplitude of diaphragm movement during tidal breathing and lung volume $(r=0.876)$. The amplitude of movement of the diaphragm with or without breathing showed no correlation to each other $(r=0.074)$. The movement during tidal breathing
\end{abstract}

shows a correlation with the changes in lung volumes. Dynamic MRI demonstrated that individuals are capable of moving their diaphragm voluntarily, but the amplitude of movement differs from person to person. In this study, the movements of the diaphragm apex and the costophrenic angle were synchronous during voluntary movement of the diaphragm while breath holding. Although the sample is small, this study confirms that the function of the diaphragm is not only respiratory but also postural and can be voluntarily controlled.

\section{Key words}

Diaphragm fiction - Magnetic resonance Paging - Thorax • Posture

\section{Corresponding author}

P. Kolár, Department of Rehabilitation, Second Medical Faculty, Charles University, Prague 5 - Motol, Czech Republic. E-mail: pavel.kolar@fnmotol.cz

\section{Introduction}

The diaphragm is generally considered to be the principal breathing muscle and as such, it is being studied and therapeutically influenced (Cluzel et al. 2000). The diaphragm function of increasing the transdiaphragmatic pressure is somewhat less studied and described. It is assumed that during diaphragmatic activity of contraction nad subsequent flattening, transdiaphragmatic pressure 
increases. Furthermore, such activity is associated with every movement of the body and extremities during force generation, including lifting or holding a load, pushing off, and applying pressure against resistance. DePalo etal. (2004) found that non-respiratory exercises (including sit-ups and bicep exercises against resistance) strengthen not only the muscles performing the movement, but also muscles used for inspiration and even expiration, with hypertrophy of the diaphragm. Similarly, Al-Bilbeisi and McCool (2000) demonstrated that weightlifters are capable of generating a greater inspiration pressure and have hypertrophied diaphragms compared to the control group.

In the current study, this cause and effect relationship of the diaphragm activity was monitored using magnetic resonance imaging (MRI) during tidal breathing and while functioning independent of respiration.

Several investigators (Gierada et al. 1995, Cluzel et al. 2000, Kiryu et al. 2006) have used MRI recording as a technique to monitor subjects' diaphragm activity. Several studies were aimed at monitoring diaphragm movement using MRI in patients with respiratory diseases or after lung surgery (Iwasawa et al. 2002, Etlik et al. 2004, Unal et al. 2000, Suga et al. 1999). However, none of these authors used MRI to monitor diaphragm movement during breath holding activities.

The current study focused on determining whether there is indeed diaphragm contraction during respiration-independent activities, and whether an individual is able to control the diaphragm voluntarily while breath holding. Diaphragm activity and its range of motion were compared during voluntary breath holding and during tidal respiration. For this study, MRI examination was used, in conjunction with evaluation of synchronized spirometric and EMG recordings of the diaphragm.

\section{Methods}

Sixteen healthy subjects were evaluated, 10 females and 6 males between the ages of 20 and 54, with a mean age of 31 years. The average weight was $71 \mathrm{~kg}$ and the average height $171 \mathrm{~cm}$. None of subjects had any pulmonary disease and/ or serious spinal complaints.

Diaphragm activity was monitored using MRI, spirometry EMG recording, in combination with the observation of ventilation using a pneumotachometer. Diaphragm activity was evaluated under two conditions: 1) during tidal breathing, and 2) while performing the exercise sequence, the patient held his or her breath at the end of the expiratory phase of tidal volume during quiet breathing.

Subjects were then instructed to pressurize and expand the abdominal wall while directing the intraabdominal pressure caudally to the pelvic floor. During this maneuver, the spine becomes stiffened and the torso is believed to become increasingly stabilized, allowing for increased resistance against internal or external loading or perturbation. The subjects were subsequently instructed to draw in the abdominal wall.

\section{MRI}

Examinations were conducted in an open MRI apparatus from Siemens, with a $0.23 \mathrm{~T}$ magnet and the software load version NUMARIS/4 syngo MR 2004A. The diaphragm was projected in the sagittal plane with the subject in a supine position, using a size large body coil. The projection plane was placed sagittally in the axial topogram directed through paravertebrally on the right side, midway through the center of the vertebral body and the edge of the thoracic wall. The width of each layer was $33 \mathrm{~mm}$. We have used true FISP dynamic sequence configured as follows: 1 NSA (number of scan acquisitions), matrix 240x256 pixels, TR $4.48 \mathrm{~ms}$, TE $2.24 \mathrm{~ms}$, flip angle $90^{\circ}$, TSE1, field of view $328 \mathrm{~mm}$. The length of each recorded sequence was $20 \mathrm{~s}$. During this time, 77 images were recorded at regular intervals; one image was acquired every $260 \mathrm{~ms}$.

Four markers, 10-ml syringes filled with water, were placed on the skin surface of each subject. They are shown as hyper signal circles on the body surface (Figs 1 and 2). The first marker was placed in the mid-clavicular line at the level of jugular opening, the second at the level of the inferior costal margin and the third at the level of the umbilicus. The last marker was placed on the subject's back at the level of the thoracolumbar junction.

\section{Spirometric recording}

Spirographic recording (volume-time during tidal breathing) was obtained using the Spirometer MasterScope Jaeger (version 5.0, Jaeger, Viasys, Wuerzburg, Germany). Tidal volume breathing was recorded by a heated pneumotachograph [Jaeger Pneumotach, with linear measurement of flow (in the range of $0 \pm 201 . \mathrm{s}^{-1}$ ) with precision $\pm 2 \%$ in the range 0.2 


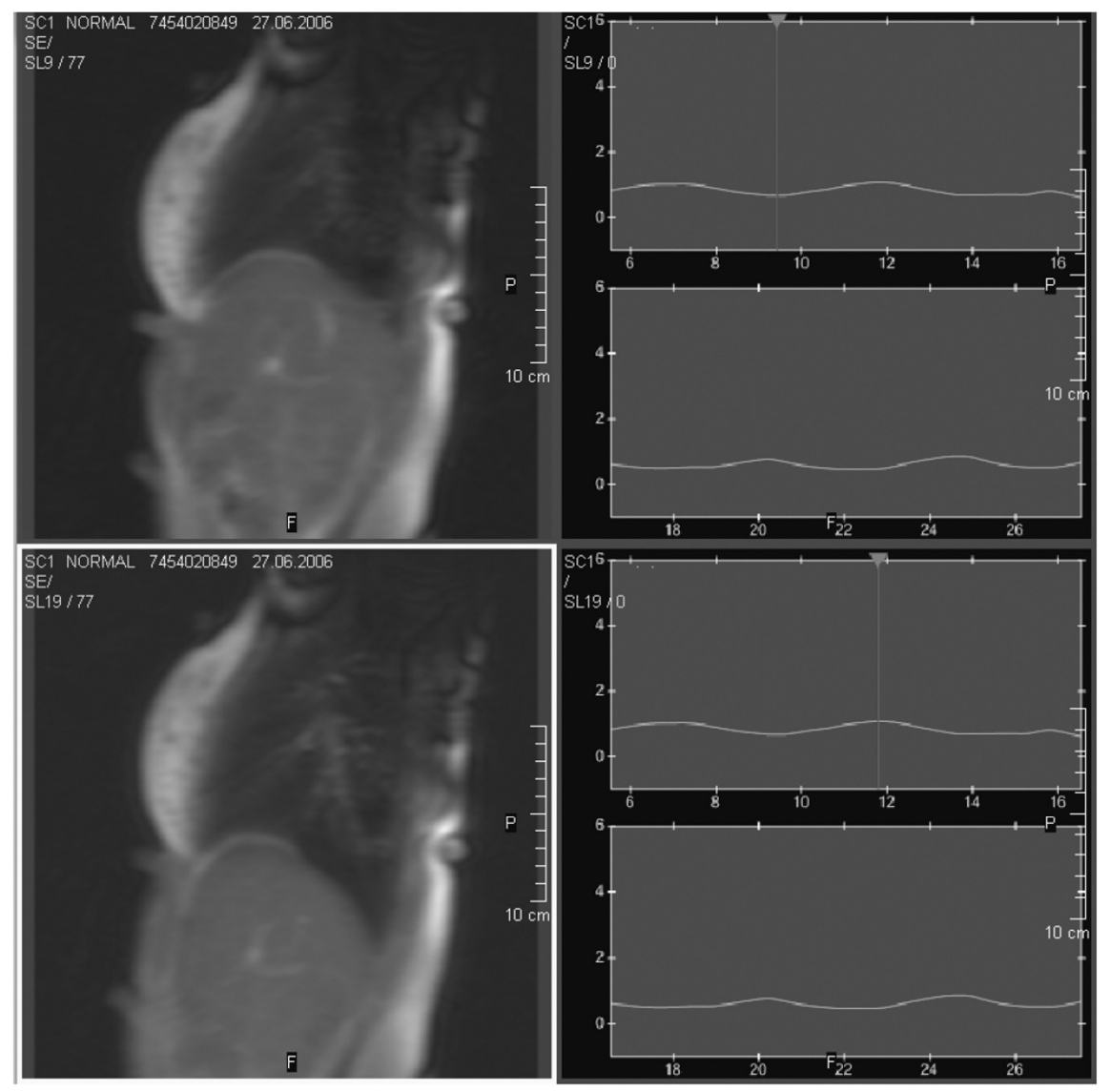

Fig. 1. MR images synchronized with spirometry of the most cranial and the most caudal position diaphragm during tidal breathing.
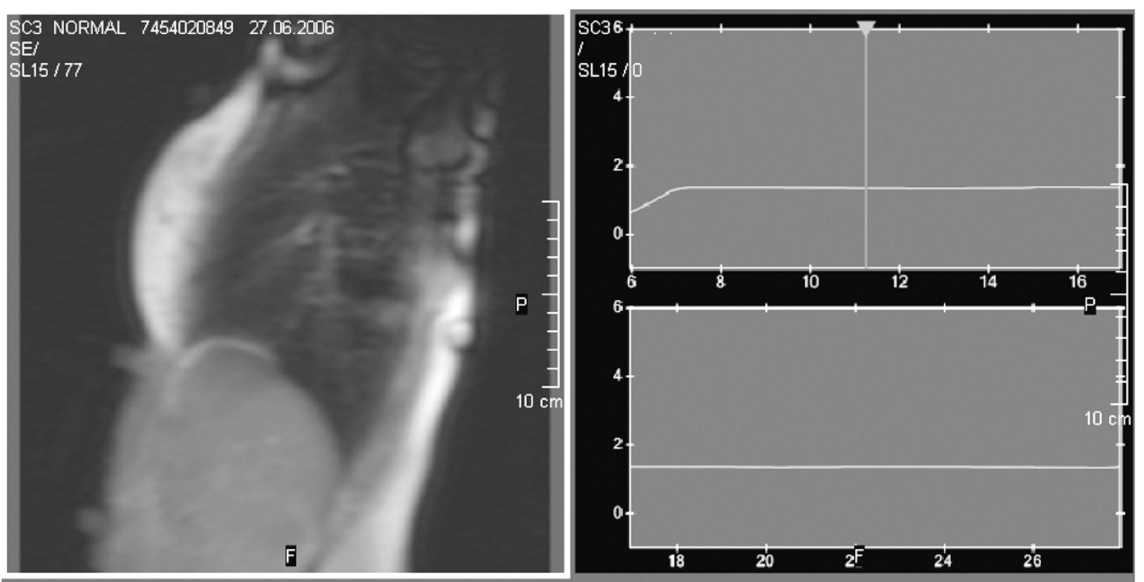

Fig. 2. $M R$ images synchronized with spirometry of the most cranial position of diaphragm during breath holding.

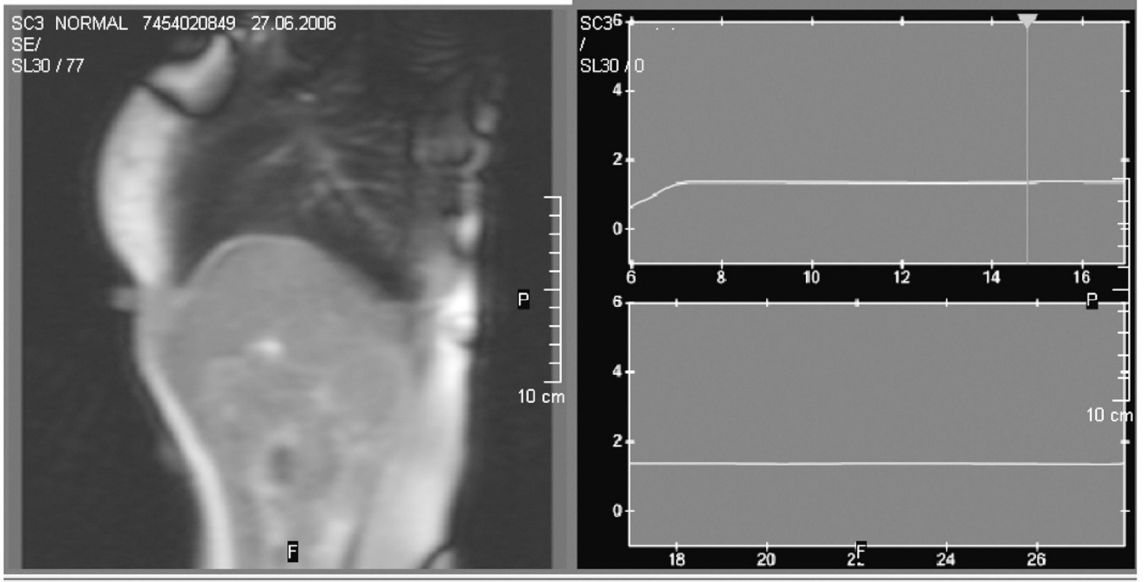



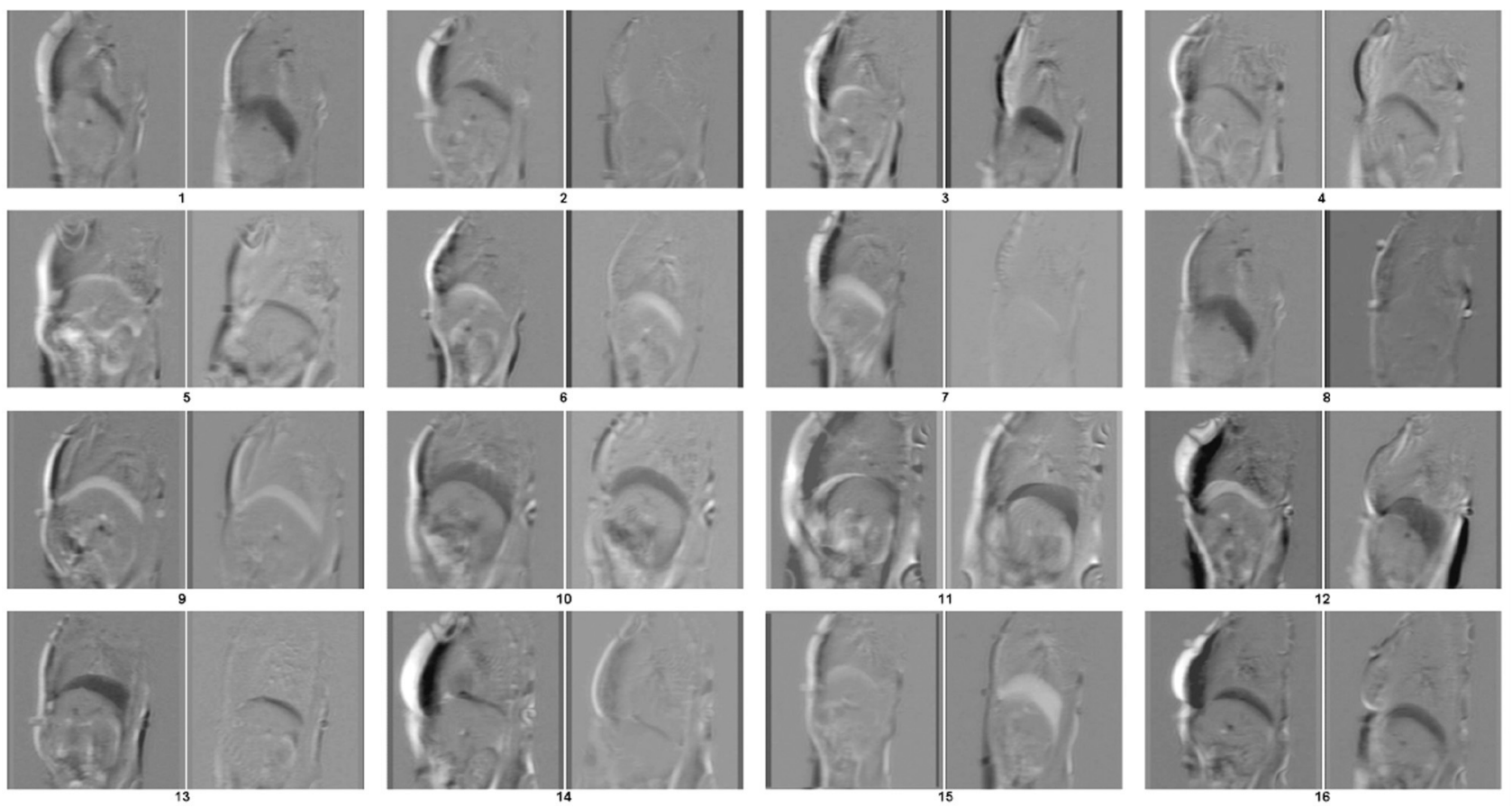

Fig 3. The subtraction of maximal and minimal positions in two sequences by the entire group of subjects.

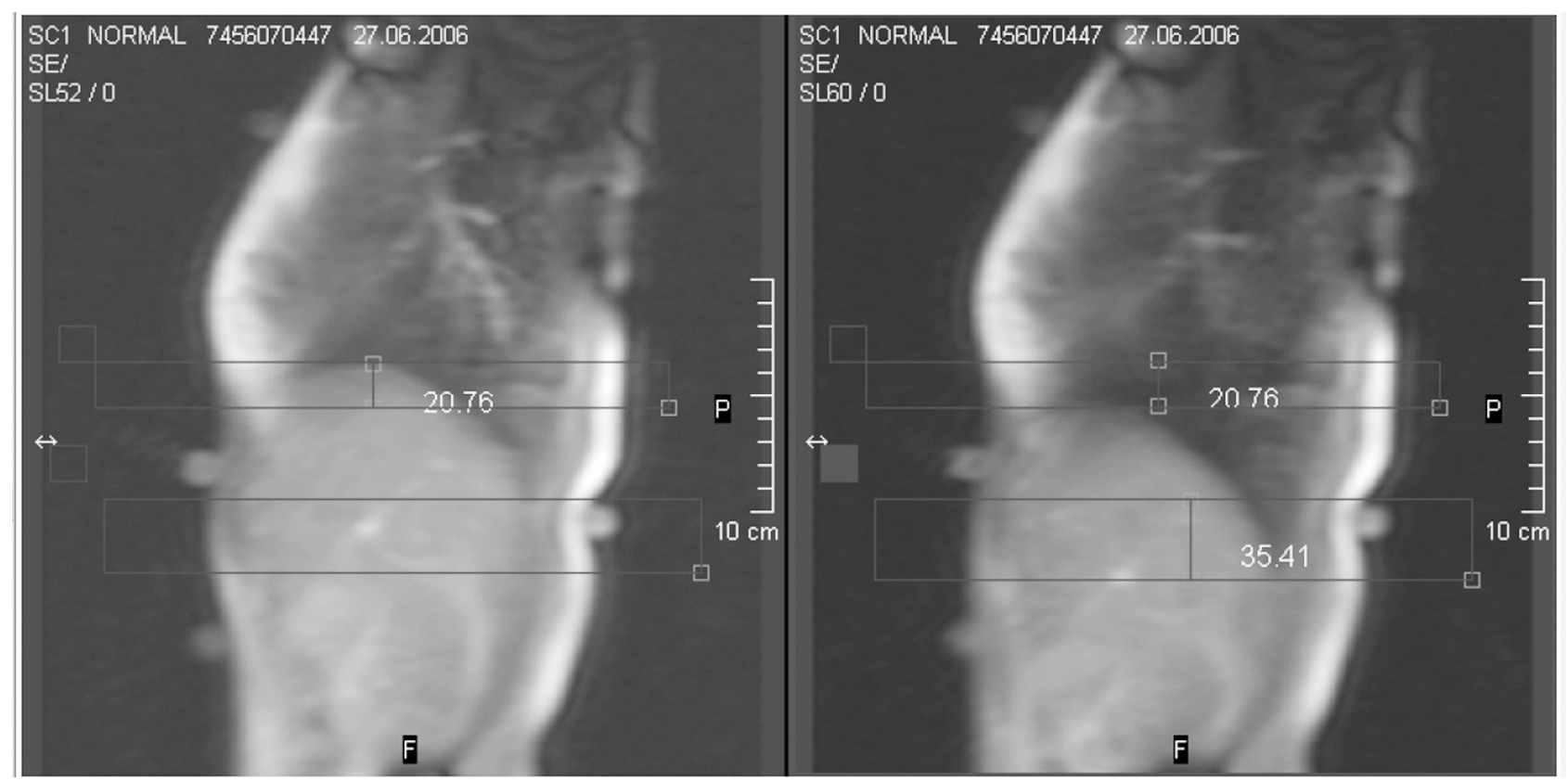

Fig. 4. Measurement of the distance between the most cranial and the most caudal position of diaphragm apex and the costophrenic angle.

to $\left.121 . \mathrm{s}^{-1}\right]$. Recording was performed using BreathRecorder (Volejník, Kurka - Jaeger Servis, s.r.o., Czech Republic), software specifically designed for this study. The values from the A-D converter were recorded to the hard drive. The values were simultaneously digitally integrated and recorded. Another support channel was recorded together with the spirometry data, which was later used to synchronize the dynamic sequence of MRI and EMG recordings. The data acquisition system was calibrated using a one liter calibration pump before each measurement. The tidal volume and the corresponding maximal and minimal superior positions of the diaphragm were calculated from the spirometry curve. 


\section{Spirometry and MR sequence synchronization}

The respiration curve recorded by the spirometer was synchronized with the beginning of the MRI recording. Individual images of spirometric curves with their relative timestamps were then converted to the DICOM compatible format and synchronized with the dynamic sequence of diaphragm movement images. Using the DICOM Scanview image monitor, the synchronized progression of the spirometric curve and the corresponding diaphragm movement were monitored.

The normal tidal breathing sequence of the diaphragm movement was compared with the sequence of breath holding. The range of motion of the diaphragm was measured and the corresponding maximal inferior and superior positions were subtracted.

\section{MRI analysis of diaphragm movement}

While recording the diaphragm movements during the tidal breathing and breath holding sequences, the maximal and minimal positions of the diaphragm were determined. Figure 1 shows the maximal inferior and superior positions of the diaphragm during tidal breathing. Graphical markings on the spirometric curve correspond to the respiration phase in which the diaphragm position was recorded on the MRI image (the curve is time-synchronized with the MRI recording).

The maximal superior and inferior positions of the diaphragm were selected from the images during the tidal breathing sequence and during breath holding (Fig. 2). These positions were selected as a reference for performing the subtractions of maximal and minimal diaphragm activation positions during breath holding. The corresponding positions of images were controlled using the contours of the torso, the contour of diaphragm and the positions of the four control markers on the body surface. The measurement was performed independently by two radiologist and in case of interobserver deviation higher than $10 \%$ consensual reevaluation was done.

As long as the most superior diaphragm position while breath holding was higher than during tidal expiration, the difference between the maximal positions was shown in white on subtracted images. If the most inferior breath holding diaphragm position was higher than during tidal inspiration, the difference between the maximal positions was shown in black on subtraction images. The subtraction of maximal and minimal positions displayed is shown in Figure 3 for the entire group of subjects.

Simultaneously, a measurement was done of the distance between the apex and posterior edge of the diaphragm and the costophrenic angle in maximal positions. This was done in such a way that a horizontal line was drawn through the edge of the diaphragm apex and the posterior costophrenic line (Fig. 4).

\section{EMG examination}

In order to prove that the recorded diaphragm movements were the result of active diaphragm contraction, an EMG analysis was performed under the same set of circumstances; during tidal breathing and during the reinforcement of the abdominal cavity (breath holding) (Fig. 5). EMG examination was performed using a concentric needle electrode and a five channel Medenec Synergy instrument. The electrode was inserted, according to Bolton, into the 7 th intercostal space along the medioclavicular line on the right. To obtain the correct EMG recording, it was necessary to precisely locate the diaphragm. While inserting the needle into the intercostalis externus muscle, which is active during inspiration, groups of MUP (motor unit potentials) of medium size were activated during inspiration. During deeper insertion, the needle passed through the intercostalis internus muscle, which is active during expiration; MUP during expiration were of medium size. Needle insertion into the diaphragm was evaluated according to two criteria. Firstly, under normal conditions, the diaphragm is activated during inspiration; secondly, the diaphragm typically has a lower, slightly narrower MUP. Using these two criteria, it was verified that the needle was positioned correctly into the diaphragm during the trials. The needle location was readjusted before each recording to satisfy these requirements. In synchrony with the EMG examination, a spirometric examination was also conducted (see the methodology of spirometric recording).

\section{Statistical evaluation}

The correlation of relative deviations in the group of 16 subjects was evaluated using a paired t-test and by correlation analysis using the Pearson's coefficient. The paired t-test was used to determine whether there was a statistically significant difference in the diaphragm range of motion (ROM) within the studied group, during tidal breathing and breath holding sequences. A correlation analysis of synchronized mobility of the posterior costophrenic angle and the diaphragm apex was performed during tidal breathing for each image sequence. The same experiment was also 

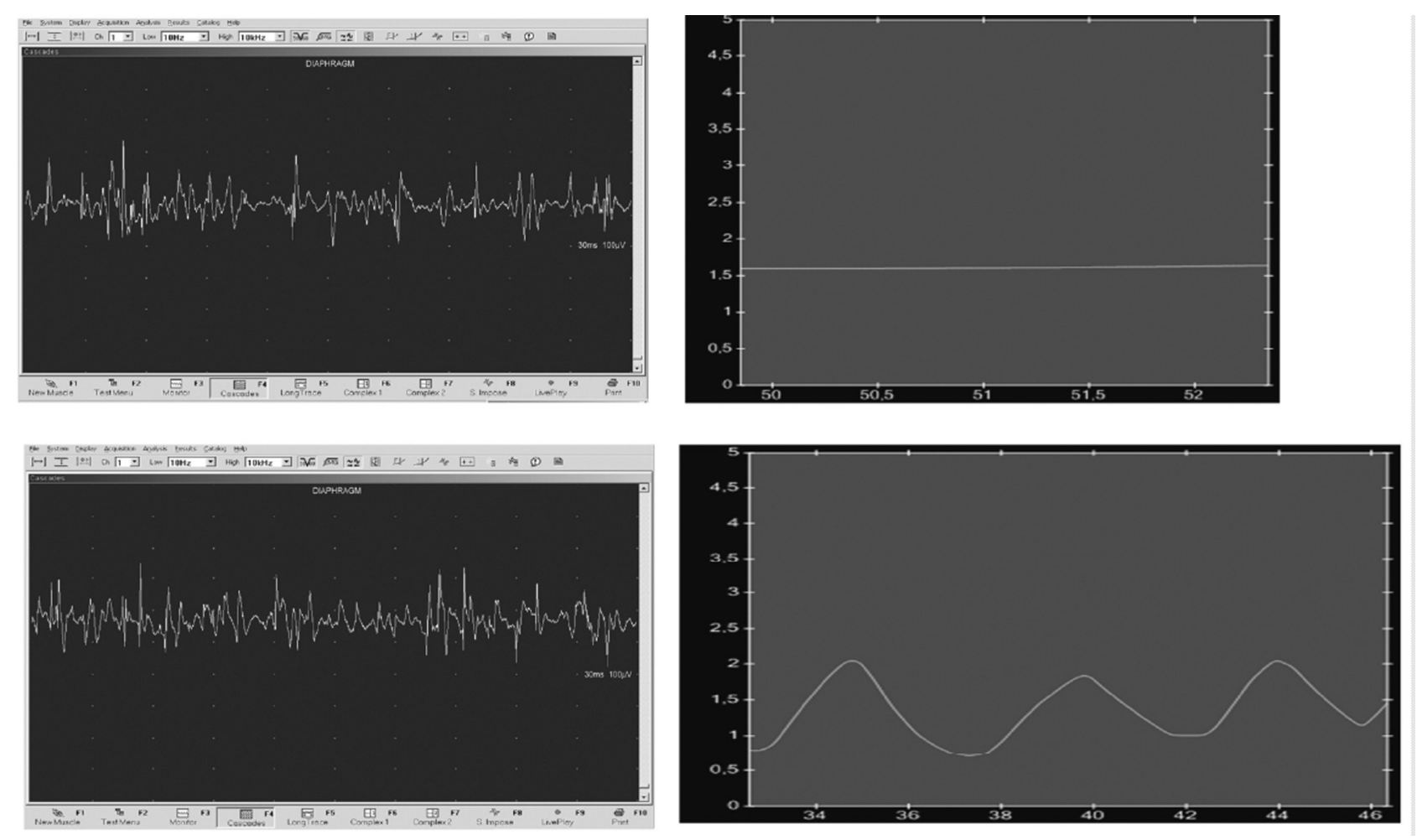

Fig. 5. EMG activity and corresponding spirometric curve.

performed while breath holding. Using correlation analysis, the synchronicity of the range of motion of the costophrenic angle and the synchronicity of the movement of the apex of the diaphragm were compared between both studied sequences in all of the 16 subjects. The correlation of the diaphragm range of motion and the lung volume was determined during tidal breathing with the use of Pearson's coefficient in 15 subjects.

\section{Results}

Among the subjects in the study group, the tidal breathing frequency and volume varied. From 2 and 4 inspiration and expiration positions of the diaphragm were observed during the 20 -s recording.

The diaphragm ROM and respiration volumes during tidal breathing were correlated. The value of Pearson's coefficient was 0.876 .

The difference after the subtraction of the maximum and minimum positions of the diaphragm during breath holding was projected in white or black color. The subtracted images of all subjects are shown on Figure 4. Similar maximal positions are evident on Figure 4 for all of the examined subjects. The maximal inferior position of the diaphragm was lower during its activation while breath holding than the inferior position during tidal inspiration in 11 of 16 subjects $(68.75 \%)$ and practically the same in 2 of 16 subjects $(12.5 \%)$. Only 3 of 16 subjects $(18.75 \%)$ showed a more inferior position during tidal breathing than during breath holding. The maximal superior position of the diaphragm during its relaxation during expiration was more pronounced than the maximal position during activation and relaxation of the diaphragm while breath holding in 7 of 16 subjects (43.75\%). It was the same in one subject and lower in 8 subjects when compared to tidal breathing.

The measured deviations, from the maximal positions of the diaphragm apex and the inferior visible edge of the costophrenic angle in the two studied cases, are displayed in the Table 1.

The diaphragm ROM (observing the positions of the diaphragm apex and the posterior costophrenic angle) during its activation while breath holding was greater than that during tidal breathing in 10 of 16 subjects. Image analysis shows a clear difference among subjects.

The average inferio-superior diaphragm apex ROM during tidal breathing was $27.3 \pm 10.2 \mathrm{~mm}$ (mean \pm SD) and during breath holding was $32.45 \pm 16.19 \mathrm{~mm}$. Movements of the visible contour of the posterior costophrenic angle were $39 \pm 17.6 \mathrm{~mm}$ during tidal breathing and $45.48 \pm 21.21 \mathrm{~mm}$ during breath holding.

T-test proved that the diaphragm ROM 
Table 1. Correlation of the diaphragm amplitude range to the respiration volume.

\begin{tabular}{|c|c|c|c|c|c|c|c|}
\hline Proband \# & $\begin{array}{l}\text { Amplitude } \\
\text { range } \\
\text { sequence } 1 \\
\text { tangent to the } \\
\text { apex } \\
(\mathrm{mm})\end{array}$ & $\begin{array}{l}\text { Amplitude } \\
\text { range } \\
\text { sequence } 1 \\
\text { posterior KF } \\
(\mathrm{mm})\end{array}$ & $\begin{array}{l}\text { Respiration } \\
\text { volume } \\
\text { sequence } 1\end{array}$ & $\begin{array}{l}\text { Amplitude } \\
\text { range } \\
\text { sequence } 3 \\
\text { apex } \\
\text { (mm) }\end{array}$ & $\begin{array}{l}\text { Amplitude } \\
\text { range } \\
\text { sequence } 3 \\
\text { posterior KF } \\
(\mathrm{mm})\end{array}$ & $(\mathrm{cm})$ & (kg) \\
\hline 1 & 12.59 & 27.47 & 0.24 & 30.90 & 46.13 & 165 & 58 \\
\hline 2 & 42.36 & 75.65 & 1.7 & 33.63 & 65.93 & 175 & 70 \\
\hline 3 & 30.14 & 45.40 & Reading error & 63.42 & 66.70 & 166 & 58 \\
\hline 4 & 18.98 & 25.56 & 0.16 & 29.00 & 36.20 & 172 & 75 \\
\hline 5 & 41.97 & 67.91 & 1.38 & 53.03 & 82.03 & 170 & 80 \\
\hline 6 & 20.76 & 36.62 & 0.65 & 10.27 & 26.99 & 163 & 73 \\
\hline 7 & 17.30 & 24.80 & 0.73 & 38.15 & 37.57 & 167 & 50 \\
\hline 8 & 30.85 & 29.76 & 1.14 & 14.35 & 21.91 & 172 & 59 \\
\hline 9 & 27.13 & 38.20 & 0.57 & 17.93 & 37.72 & 180 & 85 \\
\hline 10 & 10.68 & 8.43 & 0.32 & 20.45 & 28.56 & 178 & 90 \\
\hline 11 & 17.30 & 20.09 & 0.41 & 40.82 & 66.70 & 189 & 94 \\
\hline 12 & 20.22 & 17.17 & 0.08 & 60.67 & 83.98 & 165 & 60 \\
\hline 13 & 43.11 & 50.36 & 1.3 & 17.93 & 16.10 & 200 & 95 \\
\hline 14 & 40.22 & 49.22 & 1.22 & 46.53 & 46.77 & 168 & 74 \\
\hline 15 & 33.52 & 51.30 & 1.38 & 8.51 & 10.94 & 169 & 53 \\
\hline 16 & 29.81 & 56.20 & 0.89 & 33.64 & 53.42 & 157 & 59 \\
\hline
\end{tabular}

differences during tidal breathing compared to during breath holding, were not statistically significant. The difference in ROM of the posterior costophrenic angle of the two sequences was also insignificant.

Using correlation analysis, the movements of the posterior costophrenic angle and the apex of the diaphragm corresponded during one sequence of tidal breathing (Pearson's coefficient $\mathrm{r}=0.876$ ) and during breath holding $(\mathrm{r}=0.876)$. During comparison of the diaphragm ROM, it was demonstrated that the ROM of the diaphragm apex does not correlate during tidal breathing with the diaphragm ROM during breath holding $(\mathrm{r}=0.074)$. A minimal correlation was also discovered for the posterior costophrenic angle ROM. During tidal breathing, both the observed points moved in unison; the probability corresponding to Student t-test with paired distribution was 0.0005 . A lower level of correlation was observed during the sequence of breath holding $0.0276(\mathrm{r}=0.174)$.

The range of movement of the diaphragm top does not correlate either during the tidal breathing or during voluntarily stop breathing with the weight, the stature and the body mass index (BMI) of volunteers.
During the EMG examination, during inspiration, potentials with amplitude of less than $250 \mu \mathrm{V}$ were recorded (Fig. 5). During diaphragm activation during breath holding, potential amplitudes of less than $250 \mu \mathrm{V}$ were recorded (Fig. 5). The measurement sensitivity was $100 \mu \mathrm{V}$ per segment and time segment of $300 \mathrm{~ms}$ per screen.

\section{Discussion}

Magnetic resonance imaging was selected to record diaphragm movements for its numerous advantages compared to other methods (e.g. fluoroscopy, ultrasound); namely for the absence of radiation and the possibility to depict the whole range of diaphragm movements in multiple planes.

Gierada et al. (1995) where among the first researchers using MRI; they proved that MRI can be used to reliably record diaphragm movements. Diaphragm movement can be reliably recorded using open MRI instruments with a low magnetic field. For example, Takazakura et al. (2004) examined the diaphragm of healthy subjects using an open vertical $0.5 \mathrm{~T}$ MRI in 
horizontal and sitting positions. They analyzed the diaphragm range of motion, ROM, in the sagittal plane, observing that while sitting, the diaphragm ROM was greater, especially in its posterior section.

Chu et al. (2006) analyzed pulmonary volumes and diaphragm movements using MRI when studying adult subjects with idiopathic scoliosis and a control group of healthy subjects. They discovered that the subjects with pronounced scoliosis had a decreased diaphragm ROM as well as decreased pulmonary volumes during inspiration and expiration.

The posture stabilizing function of the diaphragm was investigated by Miyamoto et al. (2002). These authors analyzed MRI images (in the sagittal plane) for comparison of the shape and ROM of the diaphragm with and without the use of the abdominal support belt at rest, with full inspiration and with and without the Valsalva's maneuver.

Kondo et al. (2000) used MRI diaphragm images in all three planes in combination with measuring ventilation of healthy subjects using a pneumotachometer during tidal breathing and maximal deep breathing.

Takazakura et al. (2004) examined the diaphragm of healthy subjects using open vertical $0.5 \mathrm{~T}$ MRI in horizontal and seated positions. They analyzed diaphragm ROM in the sagittal plane, observing that while sitting, diaphragm ROM was greater, especially in the posterior part of the diaphragm.

Kiryu et al. (2006) compared diaphragm ROM of the left and right side of a healthy control group, while in prone, supine and side-lying positions. Diaphragm movement of the left and right side changed depending on the position, with majority of measurements of greater ROM observed on the right side.

Similar methodology to measure the diaphragm ROM was used by Takazakura et al. (2007). They studied subjects after lung resection, focusing on changes in diaphragm ROM after surgery.

None of the above authors studied the problem of explaining diaphragm activity while breath holding using MRI and EMG recordings. According to our knowledge this study is the first one showing the voluntary movement of diaphragm during the respiratory controlled breath hold. The present study focused on this aspect of diaphragm function, primarily because of its contribution to spine stabilization (by increasing intraabdominal and intrathoracic pressure) acting as an antagonist against external forces. The diaphragm contributes to spinal stabilization by flattening during which the transdiaphragmatic pressure increases. During diaphragm contraction, the spine and torso are stabilized to enable proper activation of muscles inserting on the extremities (Kolár 2006). This study was targeted towards a maneuver which takes place during each action against an external resistance (lifting of a load, push off activity etc.).

New emphasis is beginning to appear in the literature in relation to a new view on the etiopathogenesis of vertebrogenic disorders (Hodges and Gandeiva 2000). Skládal et al. (1970) has radiologically demonstrated that the diaphragm, the principal breathing muscle, participates in postural functions. Moreover, he showed that the diaphragm is associated with movement of lower extremities. Hodges and Gandeiva (2000) recorded electromyographic (EMG) activity of the diaphragm during motion of the upper extremities. An intramuscular electrode was used for EMG recording of the costal part of the diaphragm and a surface electrode was used for the EMG recordings of the deltoid and erector spinae muscles. A respiration recording of EMG activity of the diaphragm showed an increase in activity, along with an increase of EMG activity of the deltoid and erector spinae muscles. The diaphragm is only partially accessible to the application of intramuscular electrodes. The obtained measurement was thus relevant only for a part of the diaphragm and was not sufficient for a complex evaluation of its function. Even with this disadvantage, our study supports the argument that the diaphragm contributes not only to breathing but also towards the stabilization of the spine.

Hodges and Richardson (1997) and Gurfinkel (1994) mentioned that the stabilizing function of muscles, which make possible the stabilization of the pelvis and lumbar spine, appears before the beginning of movement of the upper or lower extremities. The central nervous system (CNS) must foresee the intent of movement and automatically stabilize the spine, pelvis and torso, where the activating muscles of the extremities insert (Kolár 2006). A punctum fixum, or a point of support is thus created for these muscles.

Our clinical evidence shows similar experience. While breath holding during strenuous activities, overloading of spinal segments results from insufficient and uncoordinated diaphragm activation in individuals with a weak body-stabilization function of the diaphragm. This overloading is caused by hyperactivity of superficial spinal extensors; which must compensate for this insufficiency. 
In this context an important discovery was made; the flattening of the convex contour of the diaphragm is not dependent on breathing while trying to increase the intraabdominal pressure. This capability was observed in all of the examined subjects. A wide variety of nonrespiration related diaphragm movements was found among individual subjects. The flattening of the diaphragm was more prominent or the same during breath holding, through the voluntary activation compared to the lowest position during tidal breathing in $81 \%$ examined individuals. It can therefore be inferred that there exists an individual ability to control the non-respiration activity of the diaphragm. This supports our clinical evidence that activity of the diaphragm during stabilization varies greatly among individuals. It is possible to assume that individuals with limited capability to contract the diaphragm for stabilization of the body may have a higher likelihood for development of back pain.

The results also indicate that diaphragm activation during non-respiration activity does not have to maintain the same shape during flattening, i.e. during contraction. This means that the diaphragm does not participate homogeneously as a functional unit in stabilization, but that different parts can be activated to different degrees. This supports the assumption that during EMG the recorded activity does not always correspond to the overall status of the diaphragm contraction. Further studies in this field would be helpful to verify this possibility.

This study presumes that the contour shape of the diaphragm during contraction is influenced by the interplay with the abdominal musculature. This is possibly another factor that plays an important role in explaining the results, which stem from the dysfunction of variable individual capability to use the posture stabilizing function of the diaphragm. This leads to another hypothesis that the respiratory movement of the diaphragm is synchronized with its stabilization function. It is assumed that the dysfunction of this synchronization may be one of the reasons for back pain. Confirmation of this synchronization is the subject of another study.

The relation between the ventilation parameters and the contraction activity of the diaphragm is fundamental for assessment of respiratory function. This relationship can be examined using a synchronized acquisition of spirometric data and the diaphragm MRI. The results show that the larger the difference between the expiration and inspiration diaphragm positions on the MRI images; the larger the respiration volume. The diaphragm fundamentally affects respiration volumes. It is possible to assume that pulmonary function is not only dependent on the state of the pulmonary parenchyma but also on the diaphragm range of motion. Diagnostic and therapeutic focus on the function of the diaphragm and its monitoring during the course of disease will have a fundamental significance on respiratory function. This is being confirmed especially in studies by Japanese authors, who have concentrated their efforts on monitoring diaphragm function in subjects with COPD (chronic obstructive pulmonary disease) and pulmonary emphysema (Iwasawa 2002, Etlik 2004, Unal 2000, Suga 1999).

\section{Conclusions}

The results confirm that the diaphragm is not only a breathing muscle, but is activated independently of respiration. Diaphragm movement shown on MRI images during voluntary breath holding is not only the result of pressure changes in the abdominal cavity but also of its active contraction. EMG results and the inferior diaphragm position during diaphragm contraction, which in most individuals is lower than during tidal respiration, confirm this. This finding supports the argument for the phrenic nerve's motoneuron activity being organized in such a way that the diaphragm assists more than just respiration. Non-respiration diaphragm activity is subject of voluntary control. Diaphragm ROM during both respiration and non-respiration activity differs among individuals. During tidal respiration, both the diaphragm apex and the posterior costophrenic angle move in synchrony. There is a significant correlation between the respiration volume and the diaphragm range of motion during tidal respiration.

\section{Conflict of Interest}

There is no conflict of interest.

\section{References}

Al-BILBEISI F, MCCOOL FD: Diaphragm recruitment during nonrespiratory activities. Am J Respir Crit Care Med 162: 456-459, 2000. 
CHU WC, LI AM, NG NK, CHAN DF, LAM WW, CHENG JC: Dynamic magnetic resonance imaging in assessing lung volumes, chest wall, and diaphragm motions in adolescent idiopathic scoliosis versus normal controls. Spine 31: 2243-2249, 2006.

CLUZEL P, SIMILOWSKI T, CHARTRAND-LEFEBVRE C, ZELTER M, DERENNE JP, GRENIER PA: Diaphragm and chest wall: assessment of the inspiratory pump with MR imaging-preliminary observations. Radiology 215: 574-583, 2000.

DE PALO VA, PARKER AL, Al-BILBEISI F, MCCOOL FD: Respiratory muscle strength training with nonrespiratory maneuvers. J Appl Physiol. 96: 731-734, 2004.

ETLIK O, SAKARYA ME, UZUN K, HARMAN M, TEMIZOZ O, DURMUS A: Demonstrating the effect of theophylline treatment on diaphragmatic movement in chronic obstructive pulmonary disease patients by MRfluoroscopy. Eur J Radiol 51: 150-154, 2004.

GIERADA DS, CURTIN JJ, ERICKSON SJ, PROST RW, STRANDT JA, GOODMAN LR: Diaphragmatic motion: fast gradient-recalled-echo MR imaging in healthy subjects. Radiology 194: 879-884, 1995.

GURFINKEL VS: The mechanisms of postural regulation in man. Soviet Sci Rev F. Physiol Gen Biol 7: 59-89, 1994.

HODGES PW, GANDEIVA SC: Activation of the human diaphragm during a repetitive postural task. J Physiol Lond 522: 165-175, 2000.

HODGES PW, GANDEIVA SC: Changes in intra-abdominal pressure during postural and respiratory activation of the human diaphragm. J Appl Physiol 89: 967-976, 2000.

HODGES PW, RICHARDSON CA: Relationship between limb movement speed and associated contraction of the trunk muscles. Ergonomics 40: 220-1230, 1997.

IWASAWA T, KAGEI S, GOTOH T, YOSHIIKE Y, MATSUSHITA K, KURIHARA H, SAITO K, MATSUBARA $\mathrm{S}$ : Magnetic resonance analysis of abnormal diaphragmatic motion in patients with emphysema. Eur Respir $J$ 19: 225-231, 2002.

KIRYU S, LORING H, MORI Y, ROFSKY NM, HATABU H, TAKAHASHI M: Quantitative analysis of the velocity and synchronicity of diaphragmatic motion: dynamic MRI in different postures. Magn Reson Imaging 24: 1325-1332, 2006.

KOLAR P: Facilitation of agonist-antagonist co-activation by reflex stimulation methods In: Rehabilitation of the Spine - A Practitioner's Manual. LIEBENSON C (ed), Lippincott Williams \& Wilkins, second edition, 2006a, pp 531-565.

KOLÁŘ P: Vertebrogenic complaints and stabilizing function of muscles - diagnostics (in Czech). Rehabilitace a fyzikálni lékařství 4: 155-170, $2006 \mathrm{~b}$.

KONDO T, KOBAYASHI I, TAGUCHI Y, OHTA Y, YANAGIMACHI N: A dynamic analysis of chest wall motions with MRI in healthy young subjects. Respirology 5: 19-25, 2000.

MIYAMOTO K, SHIMIZU K, MASUDA K: Fast MRI used to evaluate the effect of abdominal belts during contraction of trunk muscles. Spine 27: 1749-1755, 2002.

SKLÁDAL J, ŠKAVRAN K, KUKULENKA V: Postural function of diaphragm (in Czech). Čs Fysiol 19: 279-280, 1970.

SUGA K, TSUKUDA T, AWAYA H, TAKANO K, KOIKE S, MATSUNAGA N, SUGI K, ESATO K: Impaired respiratory mechanics in pulmonary emphysema: evaluation with dynamic breathing MRI. J Magn Reson Imaging 10: 510-520, 1999.

TAKAZAKURA R, TAKAHASHI M, NITTA N, MURATA K: Diaphragmatic motion in the sitting and supine positions: Healthy subject study using a vertically open magnetic resonance system. J Magn Reson Imaging 19: 605-609, 2004.

TAKAZAKURA R, TAKAHASHI M, NITTA N, SAWAI S, TEZUKA N, FUJINO S, MURATA K: Assessment of diaphragmatic motion after lung resection using magnetic resonance imaging. Radiat Med 25:155-163, 2007.

ÜNAL Ö, ARSLAN H, UZUN K, ÖZBAY B, SAKARYA ME: Evaluation of diaphragmatic movement with MR fluoroscopy in chronic obstructive pulmonary disease. Clin Imaging 24: 347-350, 2000. 